\title{
Etiological Study of Epistaxis in a Tertiary Care Hospital in South India
}

\author{
Dr.M.Deepthi.MS ENT, Assistant Professor ENT \\ Kamineni institute of medical sciences Narketpally,nalgonda district -508254; \\ Institute of affiliation: mgm hospital, warangal, andhra pradesh, india.
}

Key Words: Epistaxis,Etiology ,Nasal Bleeding

\section{Introduction}

Nosebleeds are reported in up to $60 \%$ of the population with peak incidences in those under the age of ten and over the age of 50 and appears to occur in males more than females[1]. An increase in blood pressure (e.g. due to general hypertension) tends to increase the duration of spontaenous epistaxis. [2]

The vast majority of nose bleeds occur in the anterior (front) part of the nose from the nasal septum. This area is richly endowed with blood vessels (Kiesselbach's plexus). This region is also known as Little's area. Bleeding further back in the nose is known as a posterior bleed and is usually due to rupture of the sphenopalatine artery or one of its branches. Posterior bleeds are often prolonged and difficult to control. They can be associated with bleeding from both nostrils and with a greater flow of blood into the mouth[1].Epistaxis is most commonly encountered in pediatric population secondary to digital trauma to kiesselbach's plexus[3] .A relationship between Hypertension and Epistaxis is a debate ,but they are most common associated finding in severe or refractory Epistaxis.[4] .Association between Heriditaryhaemorrhagic telangiectasia and Epistaxis has been determined[5].

\section{Classification Of Epistaxis}

Traditionally epistaxis has been classified based on etiology.but now most cases are found to be idiopathic , a clinical classification based on the pattern of presentation of epistaxis is more useful [6].

\section{Based on causal factors}

Primary - no causal factors (Idiopathic)

Secondary - proven causal factor

2. Based on age at presentation

Childhood - less than 16 years

Adult - more than 16 years

\section{Based on site of bleeding point}

Anterior - bleeding point anterior to pyriform aperture

Posterior - bleeding point posterior to pyriform aperture

4. Based on severity (descriptive classification)

Recurrent (usually minor and non life- threatening )

Acute severe (usually life threatening)

The cause of nosebleeds can generally be divided into two categories, local and systemic factors, although it should be remembered that a significant number of nosebleeds occur with no obvious cause(Idiopathic).

i. Congenital Causes: The common congenital causes are, Hereditary hemorrhagic telangiectasia of little's area,Osler 's disease etc [5] ,Rendu-Osler -Weber Syndrome (a triad of epistaxis, multiple mucosal and cutaneous telangiectasias), Von Willebrand disease ( factor VIII deficiency and prolonged bleeding time).

\section{ii. Acquired Cause}

Local factors

Most common factors

- Blunt trauma (blow over nose with or without a nasal bone fracture)

- Finger nail trauma.(epistaxis digitorum)

- Foreign bodies (neglected foreign body,rhinolith,maggots ,leech infestation)

- Inflammatory reaction (e.g. acute respiratory tract infections, chronic sinusitis, allergic rhinitis or environmental irritants,granulomata like syphyllis ,rhinoscleroma,tuberculosis) 
Other possible factors

- Anatomical deformities (e.g.septal spurs )

- $\quad$ Surgery (e.g.septoplasty and Functional Endoscopic Sinus Surgery)[9]

- Insufflated drugs (particularly cocaine)

- Intranasal tumors (e.g.Nasopharyngeal carcinoma or nasopharyngeal angiofibroma)

- Low relative humidity of inhaled air (particularly during cold winter seasons)

- Nasal cannula $\left(\mathrm{O}_{2}\right)$ (tending to dry the olfactory mucosa)

- Nasal sprays (particularly prolonged or improper use of nasal steroids)

- Oticbarotrauma (such as from descent in aircraft or ascent in scuba diving)

- Leech infestation

\section{Systemic Factors}

\section{Most common factors}

- Allergies

- Infectious diseases (e.g. common cold)

- Hypertension

- $\quad$ Allergic to asprin (NSAID)

\section{Other Possible Systemic Factors}

- Drugs - Aspirin, Fexofenadine/Allegra/Telfast, warfarin, ibuprofen, clopidogrel, isotretinoin, desmopressin, ginseng and others

- Alcohol (due to vasodilation)

- Anaemia

- Connective tissue disease

- Blood dyscrasias

- Hepatic failure

- Renal failure

- Envenomation by mambas, taipans, kraits, and death adders

- Heart failure (due to an increase in venous pressure)

- Hematological malignancy

- Idiopathic thrombocytopenic purpura

- Harmonal (menstruation, Pregnancy (rare) -vicarious menstruation

- Vascular disorders

- Vitamin C or Vitamin K deficiency

- von Willebrand's disease

- the consumption of hot foods and drinks e.g. sugar, salt, nuts, coke (googka) etc.

- Environmental factors -High altitude,extremes of temperature.

\section{Aims And Objectives}

1. To study the Age distribution of patients of Epistaxis referred to MGMH Warangal during the study period of $1 \frac{1}{2}$ years ie; from January 2009 to July 2010 .

2. To determine distribution of various Etiologies of Epistaxis among the study group.

3. To study Age distribution among various Etiologies.

\section{Material And Methods}

This is an Observational study wherePatients reffered to MGMH Warangalwith complaint of Epistaxis during the period of one and half year (ie; between January 2009 to June 2010) are studied for etiology .

\section{Criteria Of Selection :}

Inclusion Criteria :Random selection of patients coming to MGMH with complaints of Epistaxis, both to out patient department and Emergency department and inpatient department.

Exclusion Criteria :Patients refusing Admission or consenting for surgical intervension.

\section{Material And Methods}

Patients are examined after the initial measures to control bleeding.All admitted patients both in ENT department and Other departments with complaint of Epistaxis are subjected to routine tests like Clinical 
examination, Complete blood picture with Total leucocyte count and platelet count and Bleeding and Clotting time estimation ,Erythrocyte Sedimentation tests, Renal function tests like Blood urea and Serum creatinine ,Random blood sugar , X -Ray Paranasal sinuses, Diagnostic nasal endoscopy using 0 and 30 degree scopes ,HIV and $\mathrm{HbsAg}$ tests. Those patients with suspicion of specific cause are subjected to specific tests to diagnose the cause of bleeding like Liver function tests, Ultrasonogram of abdomen ,CT scan of Paranasalsinuses.All patients of Idiopathic and Hypertensive Epistaxis and those patients whose bleeding is not controlled by conservative measures and nasal packing are subjected to additional tests like- Coagulation profile, and if found abnormal, serum levels of specific clotting factors are estimated.Patients with tumour causing epistaxis were treated by exision of tumour and biopsy sent for histopathological examination to know the type of tumour.

\section{Results / Observations}

In the present study 200 cases of epistaxis reffered to out patient ,inpatient and casualty blocks of Mahatma Gandhi Memorial Hospital, warangal ,Andhra Pradesh ,South India, were studied during the period from January 2009 to July 2010.

I .Age And Sex Variation: (Fig I )This study shows the following observations:

Sex variation among the study group ( 200 cases $)$ includeMale $:$ Female $=146: 54=2.7: 1$.

Here the majority of cases (86) were in the age group of 21-40 emphasizing that epistaxis is a problem more common among adolescents and young adults and more common among males.

Ii .Etiology: The etiology distribution among 200 cases of present study was as follows:(Figure no .II)

A) Traumatic Cases : Out of all trauma cases( Blunt trauma- 62 and Finger Nail trauma -12), blunt trauma is more common in 21-40 year age group ie; adolescents and young adults males.Finger nail trauma is observed among 0- 20 year age group ie; among children and adolescents.(Figure no . III)

B) Idiopathic Cases: Among the study group of 200 cases , 59 cases are labelled as Idiopathic (Male : female = 45 :14). Idiopathic cases are more common among 41-60 years ie; middle age group, next common among young adults, ie; 21-40 years group (Figure no.IV).

C)Hypertensive Epistaxis: Out of 22 casesof hypertensive epistaxis,males are 18 and Females are 14 in number (Males :Females $=18: 4=4.5: 1$.Hypertension is more common in $61-80$ year age group,and among males.(Figure no.V).

D) Infections Causing Epistaxis :Out of 24patients, males and females are equally distributed.These include Acute rhinosinusitis, Vestibulitis , and Rhinosporidiosis. Among 12 patients of acute rhino sinusitis , 10 patients belong to $21-40$ year group, One patient belong to $0-20$ year age group.One patient belong to $41-60$ year group.Among 11 patients of vestibulitis, all of them belong to $0-20$ year age group.(Figure no.VI).

E) Bleeding And Coagulation Disorders : Four cases were detected,they are Post measles thrombocytopenia ,Drug induced thrombocytopenia(Ecosprin) ,Clotting factors deficiency( liver failure ), and Clotting factors deficiency .

F)Tumours :Eight of 200 cases are found to have tumors.(Table no .I) .Nasopharyngeal Angiofibroma (NPF) is found in adolescent males. Haemangiomas and inverted papilloma are found in middle age group. Maxillary tumors are found in elderly males.

G )Secondary To Dns And Septal Spurs: Seven cases are detected,among them 6 patients were male ,1 was female .More common in 21-40 year age group ie; young adults. All of them were treated conservatively initially, later after 1 week septoplasty is done.

H) Others: Two patients are categorized in this group. One of them is Rhinolith , managed by removal under general anesthesia.Other is Post septoplasty bleed ,managed by revision anterior and post nasal packing and internal maxillary artery embolisation by the interventional radiologist.

General Examination: General examination revealed rise of blood pressure in 22 patients with hypertension ,was in the range of $180-220 \mathrm{~mm} \mathrm{Hg}$ systolic and $90-130 \mathrm{mmHg}$ diastolic.Moderate to severe anemia was present in 3 patients who had recurrent and persistent bleeding for more than 3 to 4 months. The blood pressure among normotensive patients was in the range of $100-140 \mathrm{~mm} \mathrm{Hg}$ systolic and $70-90 \mathrm{~mm} \mathrm{Hg}$ diastolic.On 
systemic examination of the cardiovascular, respiratory, central nervous system and per abdomen, no abnormality was found.

Investigations:_Routine investigations were done in all cases except those of finger nail trauma and vestibulitis who are treated on Out patient department. Peripheral smear was normal in all patients.Coagulation profile and serum clotting factor estimation was done in all cases of idiopathic and hypertensive epistaxis by suspicion of bleeding or coagulation disorder.Routine hematological investigations revealed anemia in 3 patients, they are2cases of idiopathic epistaxis with anemia and one case of epistaxis secondary to clotting disorder.Platelet count was low in one patient with epistaxis secondary to post measles thrombocytopenia.Bleeding time was elevated in one patient, of post measles thrombocytopenia .Clotting time were elevated in 3 patients out of which, one patient with Von WilliBrand disease, one patient was having clotting factor deficiency secondary to liver failure .Serum albumin was decreased in the patient with epistaxis secondary to liver failure. Blood urea and sugar were within normal limits in all patients.ECG was normal in all elderly patients.X Ray PNS and X Ray Nasal Bones was done in all patients of injury nose except those of finger nail trauma. Computed Tomography (CT) of Paranasal sinuses was done in 7 patients,one patient found to have Fungal sinusitis ,2 patients had Nasopharyngeal Angiofibroma, one patient had Inverted papilloma,2 patients hadsinonasaltumours and onepatient of poly trauma with mid face fracture

\section{Discussion}

In most cases of epistaxisthe cause is unknown. Both local and systemic causes can play a role in it.GulshanHussain et al [10], concluded that "bimodal presentation with incidence peaks below 25 yrs and above 50 yrs, male $:$ female $=2.15: 1.04$, most common cause of epistaxis was found to be Trauma $(50.79 \%)$ followed by Hypertension $(22.36 \%)$. Anterior bleeding $(71.6 \%)$ was more common than posterior bleeding $(28.35 \%)$. The present study is coinciding with the above study. we found the cause to be trauma in $37 \%$, idiopathic in $29.5 \%$, hypertension in $12 \%$, Infection in $12 \%$,tumors in $4 \%$, spur bleeds in $3.5 \%$ ,bleeding diathesis in $2 \%$ and others fall in $1 \%$.Thus trauma is most common local cause.

In the same study[10], hypertension was the most common systemic cause for epistaxis, in our study also same fact is prooved . Bleeding diathesis was the next most common systemic cause following the hypertension. Epistaxis associated with the use of anticoagulants or antiplatelet drugs, was $0.5 \%$.

FIGURE .NO: I

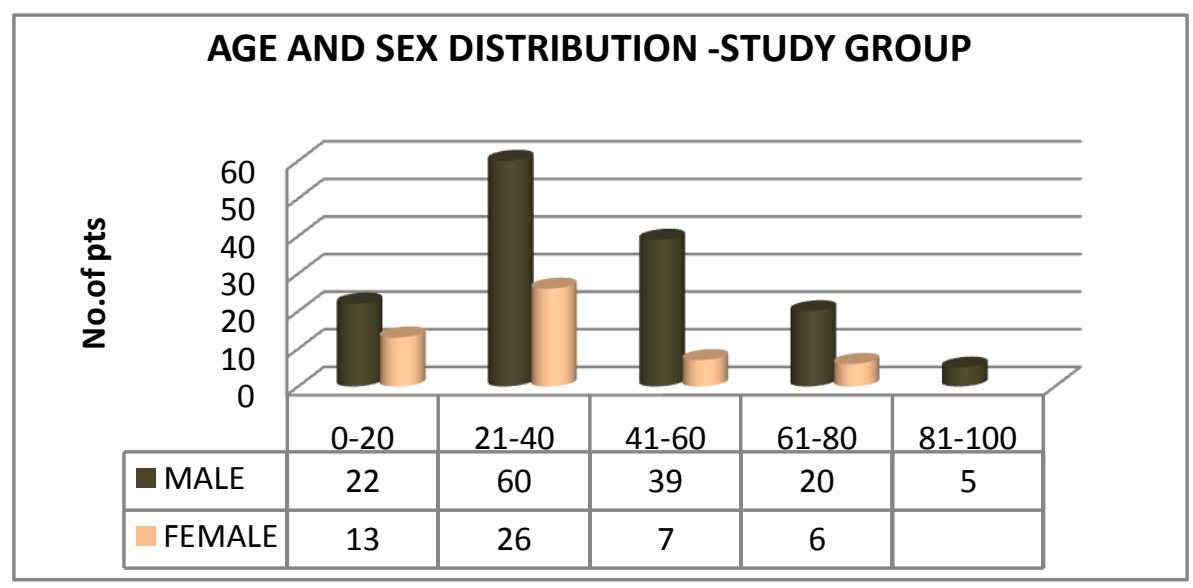

FIGURE NO: II

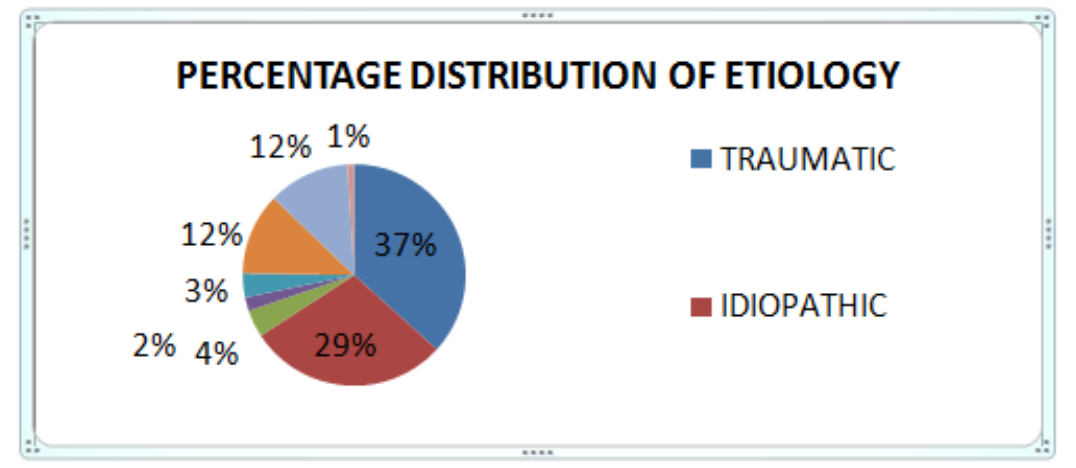


FIGURE NO: III

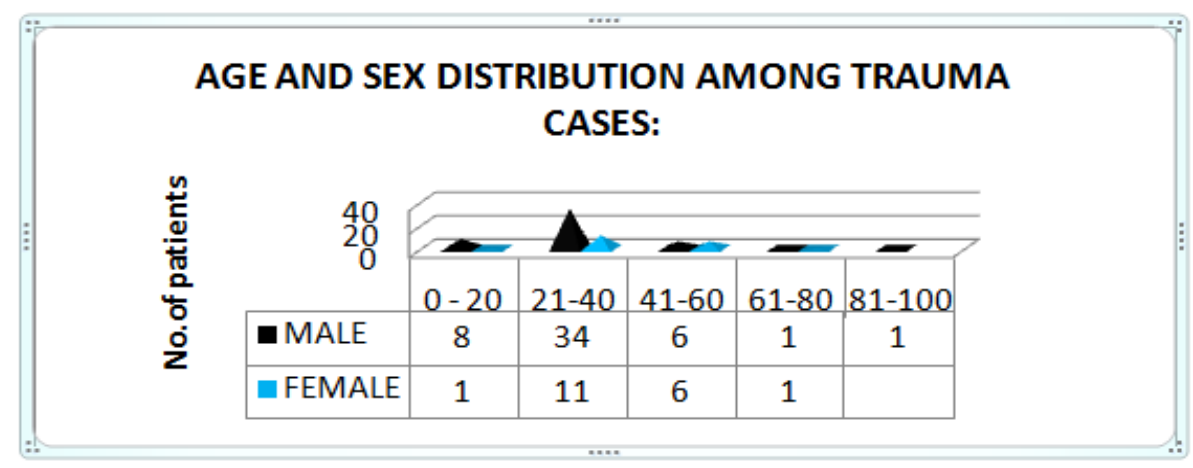

FIGURE NO: IV

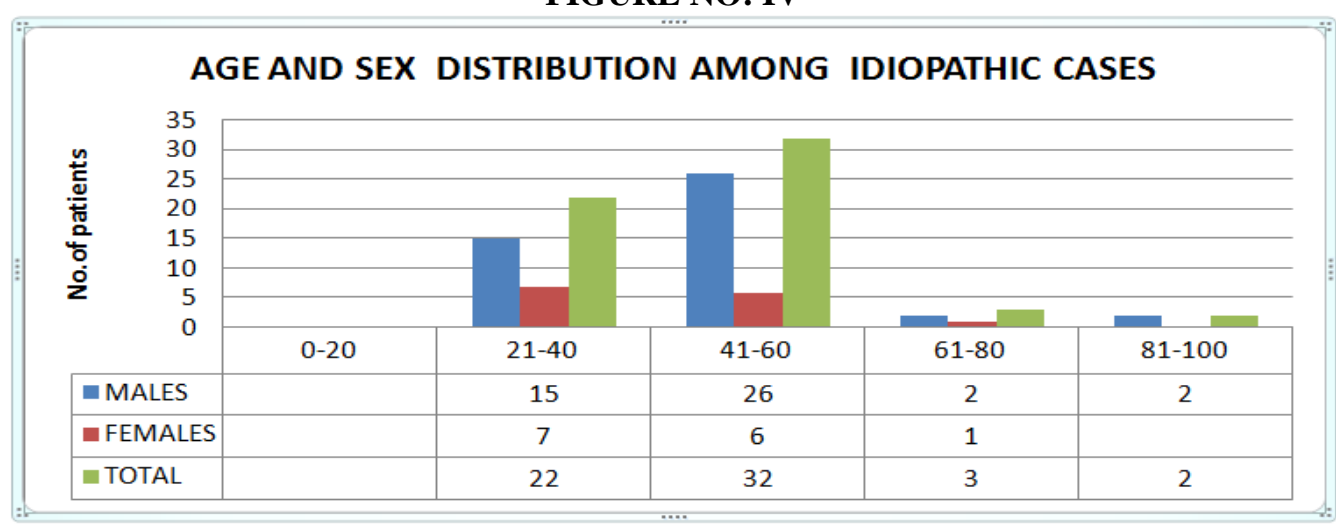

FIGURE NO: V

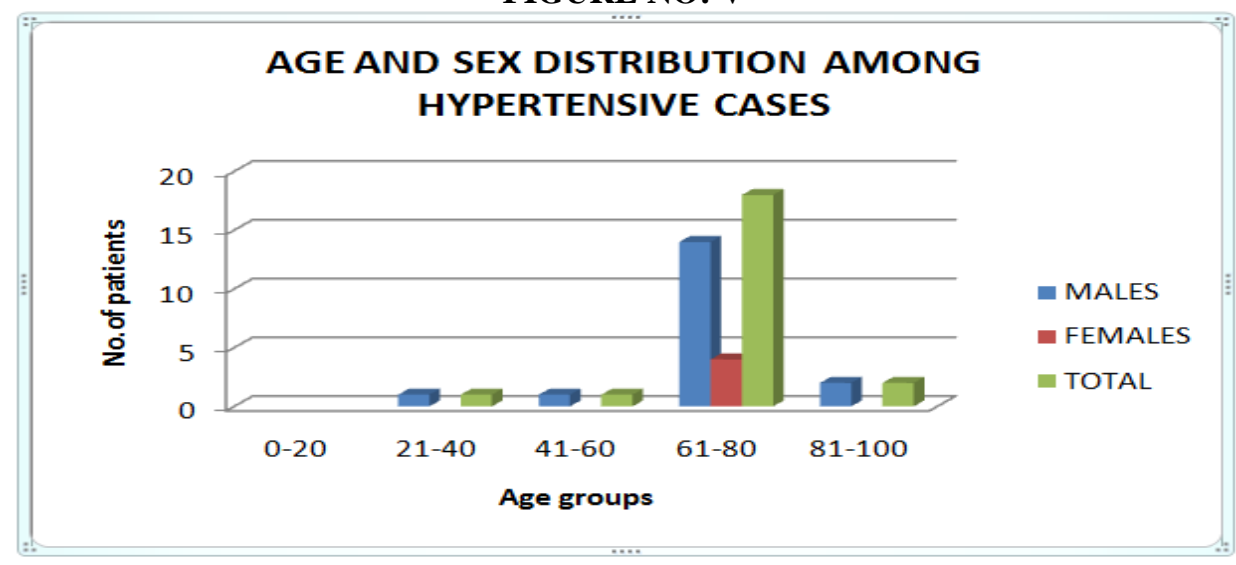

FIGURE NO: VI

\section{NO.OF PATIENTS WITH INFECTIONS - AGE AND SEX DISTRIBUTION}

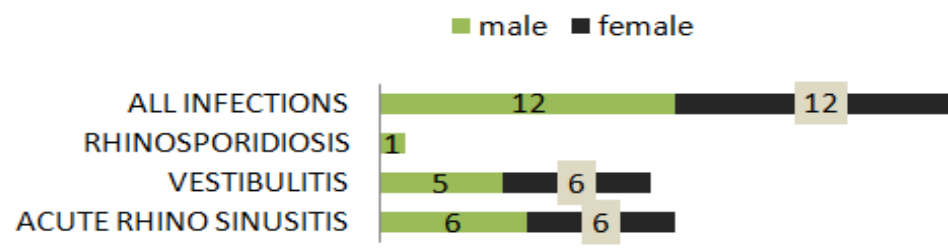


TABLE NO: I

No .Of Patients And Sex Distribution Among Patients With Tumors

\begin{tabular}{|l|l|l|l|}
\hline TUMOUR TYPE & MALE & FEMALE & TOTAL \\
\hline Nasopharyngeal Angiofibroma & 2 & - & 2 \\
\hline Haemangiomas & 2 & 1 & 3 \\
\hline $\begin{array}{l}\text { Maxillary sinonasaladeno } \\
\text { carcinoma }\end{array}$ & 2 & - & 2 \\
\hline Inverted papilloma & 1 & - & \\
\hline
\end{tabular}

VI. Summary

In the present study, study group of 200 patients are choosen randomly from out patient and inpatient and casualty departments of MGMH Warangal with complaint of Epistaxis . Age and sex distributions among various etiologies of Epistaxis was studied and it was found to be more common in males when compared to females $($ male $:$ female $=2.7: 1$ ). Trauma was found to be the most common etiology $(37 \%)$, more in young adult males (21-40 years). Next common causeis, idiopathic. Fingernail trauma is more common in male children. Among middle age group ,Idiopathic and trauma are common .Among elderly, Hypertension was more common in 61-80 years age group. The site of bleeding was undetermined in a large majority of patients even after proper nasal endoscopy.Our study supports the view that the history of use of any anticoagulant or aspirin use and also history of recurrent epistaxis and bleeding from other sites of the body must be elicited in all the patients presenting with epistaxis. Routine investigations must include coagulation profile, liver function tests and renal function tests for every case of epistaxis. Treatment of severe epistaxis can encompass many modalities depending upon its site, severity and etiology.

\section{References}

[1]. Corry J. Kucik\& Timothy Clenney (January 15, 2005). "Management of Epistaxis". American Academy of Family Physicians. http://www.aafp.org/afp/20050115/305.html. Retrieved January 31, 2010.

[2]. J. F. LubiancaNeto, F. D. Fuchs, S. R. Facco, M. Gus, L. Fasolo, R. Mafessoni\& A. L. Gleissner (1999). "Is epistaxis evidence of end-organ damage in patients with hypertension?". Laryngoscope 109 (7): 1111-1115. PMID 10401851.

[3]. Cummings C.W. Cummings Otolaryngology Head and Neck Surgery. $4^{\text {th }}$ ed.Philadelphia,PA;Elsevier Mosby;2005.

[4]. GuariscoJ.L.,Graham H.D ${ }^{\text {rd }}$.Epistaxis in children :Causes ,diagnosis and treatement .Ear Nose and Throat J.1989;68(7)[ PubMed]

[5]. SharathkumarA.A.,ShapiroA.Hereditaryhaemorrhagic telangiectasia.Haemophilia.2008;14(6):1269-1280.[PubMed]

[6]. Gerald W McGarry, Scott-Brown's Otorhinolaryngology, Head and Neck Surgery, 7th Edition,VOL.2-A; Part 13,126; 1596 $-1599,1603-1604$

[7]. Shaheen O.H: "Epistaxis" in Scott - Brown's otolaryngology, volume 4, 6th edition, 278 - 282; 1987.

[8]. Valerie J Lund and Geoffrey E Rose, Scott-Brown's Otorhinolaryngology, Head and Neck Surgery, 7th Edition,VOL.2-A; Part 13,$132 ; 1678$

[9]. Mayer D.M; Swanker W.A: postoperative nasal bleeding. Archives of otolaryngology. 54, 384-389; 1951.

[10]. GulshanHussain et al ,Evaluation of aetiology and efficacy of management protocol of epistaxis ,J Ayub Med Coll Abbottabad 2006; 18 